\title{
Alternativas microbiológicas para la remediación de suelos y aguas contaminados con fertilizantes nitrogenados
}

\author{
Microbiological alternatives for the remediation of soils and water contaminated with \\ nitrogen fertilizers
}

\author{
L. A. García-Galindo ; A. Capera-Rivas ; J. P. Melendez iD ; N. Mayorquín iD
}

\begin{abstract}
Resumen - El Nitrógeno (N) es considerado el nutriente más importante para la producción vegetal, por lo que se requiere fertilizar con cantidades del mismo que permitan garantizar el adecuado desarrollo de los cultivos. Actualmente, los fertilizantes de síntesis química con amonio $\left(\mathrm{NH}_{4}{ }^{+}\right)$, nitrato $\left(\mathrm{NO}_{3}{ }^{-}\right)$o úrea $\left(\mathrm{CO}\left(\mathrm{NH}_{2}\right)_{2}\right)$, son los más utilizados; sin embargo, se dosifican y aplican a los suelos de forma incorrecta empleando cantidades elevadas con poca eficiencia, causando problemas de salinidad, toxicidad y de contaminación en suelos y aguas, lo que a su vez tiene efectos en los ecosistemas y la salud. Teniendo en cuenta la necesidad de continuar generando tanto productos, como mecanismos, que permitan garantizar la productividad de los sistemas agrícolas y su aporte a la economía y seguridad alimentaria de los países de forma sostenible, se han planteado soluciones variadas al respecto. En este escrito se buscó analizar los efectos generados por los fertilizantes nitrogenados en los suelos y aguas, así como las alternativas planteadas por varios autores que permiten minimizar el impacto de estos compuestos en el ambiente. Tras realizar una revisión sistemática de diversos estudios sobre el tema, se observó que en la literatura de los últimos 20 años se resalta el uso de microorganismos para la biorremediación, así como la aplicación de biofertilizantes como las alternativas más relevantes y eficientes para contrarrestar los efectos contaminantes con fertilizantes nitrogenados de forma sostenible.
\end{abstract}

Palabras claves - Biofertilizantes; Biorremediación; Contaminación hídrica, Nitrógeno $(\mathrm{N})$, Suelos agrícolas.

Abstract-Nitrogen (N) is the most important nutrient for plant production and therefore it is necessary to fertilize with quantities of it that allow ensuring the proper development of crops. Currently, chemical synthesis fertilizers containing ammonium $\left(\mathrm{NH}_{4}{ }^{+}\right)$, nitrate $\left(\mathrm{NO}_{3}{ }^{-}\right)$or urea $\left(\mathrm{CO}\left(\mathrm{NH}_{2}\right)_{2}\right)$, are the most commonly used. However, the way in which they are dosed and applied to soils is not suitable, since they are used in high quantities with little efficiency, generating a high environmental impact by causing

Este manuscrito fue enviado el 21 de agosto de 2019 y aceptado el 08 de marzo de 2020. Este trabajo se realizó con el apoyo de la Facultad de Ciencias Básicas y el programa de Procesos Ambientales de la Fundación Universitaria Horizonte, al interior del Semillero de Estudios Ambientales (SEAmbiental)

L. A. García-Galindo, docente e investigadora de la Facultad de Ciencias básicas de la Fundación Universitaria Horizonte, Bogotá, Colombia (e-mail: luiagarciagal@unal.edu.co)

A. Capera-Rivas, estudiante del Técnico Profesional en Procesos Ambientales de la Fundación Universitaria Horizonte, Bogotá, Colombia (email: arzaled_capera@unihorizonte.edu.co) salinity, toxicity and pollution problems in soils and water, which in turn has effects on ecosystems and health. Recognizing this problem, and taking into account the need to continue generating both products and mechanisms, that allow to guarantee the productivity of agricultural systems and their contribution to the economy and food security of countries in a sustainable way, they have been raised varied solutions in this regard. This paper sought to analyze the effects generated by nitrogen fertilizers in soil and water, as well as the alternatives proposed by several authors that minimize the impact of these compounds on the environment.After conducting a systematic review of various studies on the subject, it it was observed that in the literature of the last 20 years, the use of microorganisms for bioremediation is highlighted, as well as the application of biofertilizers as the most relevant and efficient alternatives to counteract in a sustainable way the contaminating effects with nitrogen fertilizers.

Index Terms-Agricultural Soils, Biofertilizers, Bioremediation, Nitrogen (N), Water Pollution.

Resumen - El Nitrógeno $(\mathrm{N})$ es considerado el nutriente más importante para la producción vegetal, por lo que se requiere fertilizar con cantidades del mismo que permitan garantizar el adecuado desarrollo de los cultivos. Actualmente, los fertilizantes de síntesis química con amonio $\left(\mathrm{NH}_{4}{ }^{+}\right)$, nitrato $\left(\mathrm{NO}_{3}{ }^{-}\right)$o úrea $\left(\mathrm{CO}\left(\mathrm{NH}_{2}\right)_{2}\right)$, son los más utilizados; sin embargo, se dosifican y aplican a los suelos de forma incorrecta empleando cantidades elevadas con poca eficiencia, causando problemas de salinidad, toxicidad y de contaminación en suelos y aguas, lo que a su vez tiene efectos en los ecosistemas y la salud. Teniendo en cuenta la necesidad de continuar generando tanto productos, como mecanismos, que permitan garantizar la productividad de los sistemas agrícolas y su aporte a la economía y seguridad alimentaria de los países de forma sostenible, se han planteado soluciones variadas al respecto. En este escrito se buscó analizar los efectos generados por los fertilizantes nitrogenados en los suelos y aguas, así como las alternativas planteadas por varios

J. P. Melendez, estudiante del Técnico Profesional en Procesos Ambientales de la Fundación Universitaria Horizonte, Bogotá, Colombia (e-mail: jean_melendez@unihorizonte.edu.co)

N. Mayorquín, estudiante del Técnico Profesional en Procesos Ambientales de la Fundación Universitaria Horizonte, Bogotá, Colombia (e-mail: nicolas_mayorquin@unihorizonte.edu.co) 
autores que permiten minimizar el impacto de estos compuestos en el ambiente. Tras realizar una revisión sistemática de diversos estudios sobre el tema, se observó que en la literatura de los últimos 20 años se resalta el uso de microorganismos para la biorremediación, así como la aplicación de biofertilizantes como las alternativas más relevantes y eficientes para contrarrestar los efectos contaminantes con fertilizantes nitrogenados de forma sostenible.

Palabras claves- Biofertilizantes, Biorremediación, Contaminación hídrica, Nitrógeno (N), Suelos agrícolas.

Resumo - O Nitrogênio (N) é considerado o nutriente mais importante para a produção vegetal, sendo necessário fertilizar com quantidades que garantam o desenvolvimento adequado dos cultivos. Atualmente, os fertilizantes de síntese química com amônio $\left(\mathrm{NH}_{4}^{+}\right)$, nitrato $\left(\mathrm{NO}_{3}^{-}\right)$ou uréia $\left(\mathrm{CO}\left(\mathrm{NH}_{2}\right)_{2}\right)$ são amplamente utilizados, porém são dosados e aplicados incorretamente no solo, utilizando grandes quantidades com baixa eficiência, causando problemas de salinidade, toxicidade e contaminação em solos e águas, o que, por sua vez, afeta os ecossistemas e a saúde. Levando em conta a necessidade de continuar gerando produtos e mecanismos que garantam a produtividade dos sistemas agrícolas e sua contribuição para a economia e a segurança alimentar dos países de maneira sustentável, várias soluções foram propostas. Neste artigo, foram analisados os efeitos gerados por fertilizantes nitrogenados em solos e águas, bem como as alternativas propostas por vários autores que permitem minimizar o impacto desses compostos no meio ambiente. Após uma revisão sistemática de vários estudos, observou-se que na literatura dos últimos 20 anos se destaca o uso de microrganismos para biorremediação, bem como a aplicação de biofertilizantes como as alternativas mais relevantes e eficientes para combater os efeitos contaminantes com fertilizantes nitrogenados de forma sustentável.

Palavras-chave Biofertilizantes, Biorremediação, Contaminação da água, Nitrogênio (N), Solos agrícolas

\section{INTRODUCCIÓN}

$\mathrm{E}$ L Nitrógeno $(\mathrm{N})$ es considerado el nutriente más importante para la producción vegetal debido a las cantidades requeridas por los cultivos y por la frecuencia con que se observan deficiencias en suelos agrícolas [1].

Sin embargo, su forma de aplicación y de dosificación no es la correcta ya que se emplea en cantidades muy altas con poca eficiencia [2,3], generando problemas de salinidad en los suelos y de contaminación tanto en suelos como en cuerpos de agua cuando los excedentes son lavados [1] debido a que dichos fertilizantes pueden contener amonio $\left(\mathrm{NH}_{4}{ }^{+}\right)$, nitrato $\left(\mathrm{NO}_{3}{ }^{-}\right) \mathrm{o}$ úrea $\left(\mathrm{CO}\left(\mathrm{NH}_{2}\right)_{2}\right)$ los cuales, en mayor o menor medida, se movilizan ocasionando que estas especies químicas, (especialmente los nitratos) afecten las propiedades generales de los ecosistemas desbalanceándolos [4].

Al reconocer esta problemática, y teniendo en cuenta la necesidad de continuar generando tanto productos, como mecanismos que permitan garantizar la productividad de los sistemas agrícolas y su aporte a la economía y seguridad alimentaria de los países de forma sostenible y ambientalmente adecuada, se han planteado desde las ciencias básicas y aplicadas, soluciones variadas al respecto dentro de las que se resaltan en la literatura, el uso de microorganismos, especialmente bacterias y microalgas, como biofertilizantes y para la biorremediación de ambientes contaminados.

Teniendo en cuenta lo anterior, el presente documento busca analizar los efectos generados por los fertilizantes nitrogenados en los suelos y aguas, así como las alternativas en las que se emplean microorganismos, que han sido planteadas como eficientes y que permiten minimizar el impacto de estos compuestos en el ambiente.

\section{METODOLOGÍA}

\section{A. Tipo de Revisión}

Se realizó una revisión sitemática (RS) de literatura de artículos y publicaciones nacionales e internacionales de acuerdo a lo planteado por García-Perdomo [5], quien señala que para que una revisión se considere sistemática, debe contar con la evaluación ordenada y explícita de la literatura a partir de una pregunta clara de investigación, basada en una estrategia clara y reproducible para dicha búsqueda, que permira la seleeción apropiada de estudios junto con la extracción de sus datos, para llegar a un análisis crírico de acuerdo a diferentes herramientas. Otros autores señalan que las RS al estar constituídas por múltiples artículos y fuentes de información aportan un alto nivel de evidencia a lo que se conoce de un tema determinado y coinciden en que para estas se debe describir el proceso de elaboración transparente y comprensible para recolectar, seleccionar, evaluar críticamente y resumir toda la evidencia disponible sobre el tema. [6]

\section{B. Fuentes empleadas}

Se indagó principalmente en bases de datos indexadas como Scopus, Sciencie direct, Google scholar, Scielo, Redalyc, y Dialnet. Se seleccionaron como fuente de información artículos de revistas indexadas, principalmente en de alto impacto, dando mayor relevancia a fuentes primarias, aunque se incluyeron en la búsqueda fuentes secundarias como revisiones de tema para nutrir la información base del estudio.

\section{Criterios de búsqueda}

Para la consulta se emplearon términos del Thesaurus "Nitrogen fertilization" acompañado del subdescriptor "nitrogen pollution". Tambien se emplearon "fertilizer AND biorremediation", “nitrogen AND biofertilizer". Estos motores de búsqueda se emplearon en inglés y en español.

Adicionalmente, se tuvo en cuenta que los artículos y/o literatura consultada correspondiera como máximo a los últimos 30 años, para lo cual se emplearon los filtros por fecha que ofrecen las bases de datos.

\section{IMPORTANCIA DE LOS FERTILIZANTES}

\section{A. Definición e historia de los fertilizantes}

Los fertilizantes se han empleado desde hace más de 5000 años, siendo en un inicio el humus el más utilizado, ya que esas materias orgánicas descompuestas, o todavía en fase de 
descomposición, eran fuente primordial para la nutrición de las plantas [7]. Posteriormente, en los 70s, tras la revolución verde generada en los Estados Unidos y que después se extendió a varios países, entre ellos Colombia, primó el crear nuevas tecnologías que proporcionaran mayor productividad por unidad de superficie [8], por lo que la revolución verde prácticamente sepulto los métodos de agricultura tradicional y le dio paso al uso de moléculas sintéticas como fertilizantes, plaguicidas y semillas híbridas; productos que han dañado el equilibrio del medio ambiente y de la salud del hombre [9].

En general, los fertilizantes son definidos como cualquier tipo de sustancia orgánica o inorgánica que contiene nutrientes en formas asimilables por los organismos vegetales, que permite mantener o incrementar el contenido de estos elementos en el suelo, mejorar la calidad del sustrato a nivel nutricional y estimular el crecimiento vegetativo de las plantas [10].

Lo anterior, enfatiza en la idea del uso de sustancias de origen natural con la finalidad de garantizar una adecuada nutrición vegetal, sin embargo, la razón por la que los fertilizantes sintéticos de origen químico son tan empleados, se debe a que aumentan el rendimiento y el potencial de producción de los cultivos en cifras difíciles de alcanzar con sólo enmiendas orgánicas [11]. No obstante, su aplicación y adecuada dosificación rara vez es estudiada, generando excedentes en suelos y aguas que repercuten en la calidad química de los mismos contaminándolos.

\section{B. Importancia de los fertilizantes nitrogenados}

De los 17 elementos esenciales para el crecimiento y desarrollo vegetal, el $\mathrm{N}$ es considerado el más importante $[1,11]$. Esto se debe a que es, luego del carbono, el elemento que se encuentra en mayor proporción en las células vegetales en las que posee funciones de tipo estructural haciendo parte de proteínas, ácidos nucleicos, clorofilas y alcaloides; y función osmótica asociada a los efectos del nitrato y a otras formas reducidas de $\mathrm{N}$, en la disminución del potencial hídrico $(\Psi \omega)$ de la vacuola, en el proceso de osmoregulación al permitir retener en estos organelos agua [12,13]. Por estas razones, los fertilizantes nitrogenados son los principales promotores del aumento en el rendimiento en la agricultura actual y su deficiencia es fácilmente detectable al observarse en poco tiempo cambios en el color y turgencia de las hojas (Fig. 1).

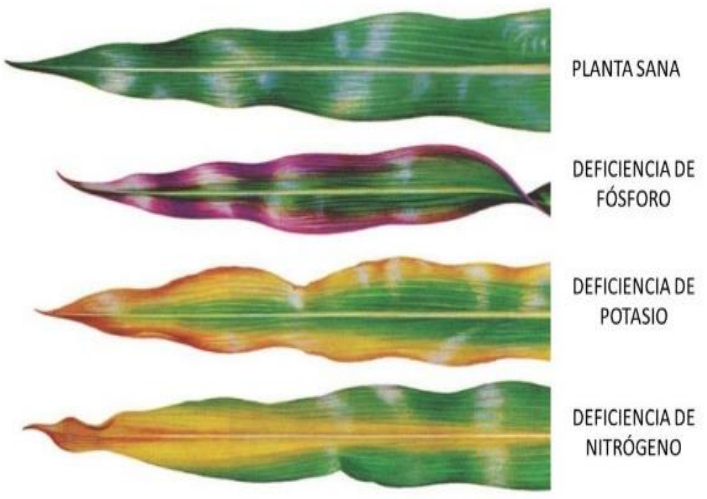

Fig. 1. Síntomas de carencia de diferentes nutrientes. [14]

Conociendo su importancia, la aplicación global de fertilizantes nitrogenados se ha venido incrementando con los años, siendo Estados Unidos, Europa, el norte de India y el este de China, con dosis de alrededor de $200 \mathrm{~kg} / \mathrm{Ha}$, los mayores consumidores [15]; al mismo tiempo que, países de vocación agrícola como los de Latinoamérica han aumentado los consumos de fertilizantes nitrogenados desde ya hace varios años.

Por ejemplo, entre 1990-2000, Argentina, incrementó el consumo de fertilizantes nitrogenados en la alarmante cifra de 27\% anual [16]. Por su parte, en México, la producción y uso de fertilizantes nitrogenados data de 1915 , y se ha establecido que de acuerdo a lo tecnificado que sea el proceso o la zona agrícola, se emplean los fertilizantes sólidos (zonas con menos recursos) o líquidos y gases (zonas más tecnificadas); observando que los de mayor consumo son los sólidos [2, 17].

En tanto, para el caso puntual de Chile, el investigador Parodi [15], señala que más allá de existir una relación entre la industria agrícola destinada a la producción de alimentos para consumo humano, los incrementos en fertilización nitrogenada están estrechamente relacionados con la fertilización de pastos para ganadería extensiva.

En Colombia, el porcentaje de producción de fertilizantes de síntesis, incluyendo los nitrogenados, ha disminuido con los años, mostrando su mayor pico en 2004 (931 millones de Ton producidas), año a partir del cual se ha mantenido estable alrededor de los 220 millones de Ton/año. Esta cifra en un inicio alentadora, se contrarresta con los valores de consumo de fertilizantes $(\mathrm{kg} / \mathrm{Ha})$, los cuales han aumentado [18] como se muestra en la Fig. 2.

Los principales abonos nitrogenados de síntesis química son la úrea, el nitrato amónico, el amoníaco y el sulfato amónico; para cada uno de los cuales debería ser calculada la dosis óptima de Nitrógeno por su diferencia química. Para determinar la cantidad de $\mathrm{N}$ se deben tener en cuenta tres factores: a) el cultivo, b) la "fertilidad" de la parcela en el momento de realizar la aplicación y c) el objetivo en términos de productividad y 
rendimiento deseados; sin embargo, por lo general estos cálculos no se realizan de forma adecuada [19], e incluso se omiten, aplicando en exceso este nutriente, pues los agricultores asumen que, como se mencionó, una considerable proporción de los fertilizantes se perderá debido a la movilidad de estos compuestos en aguas y suelos.

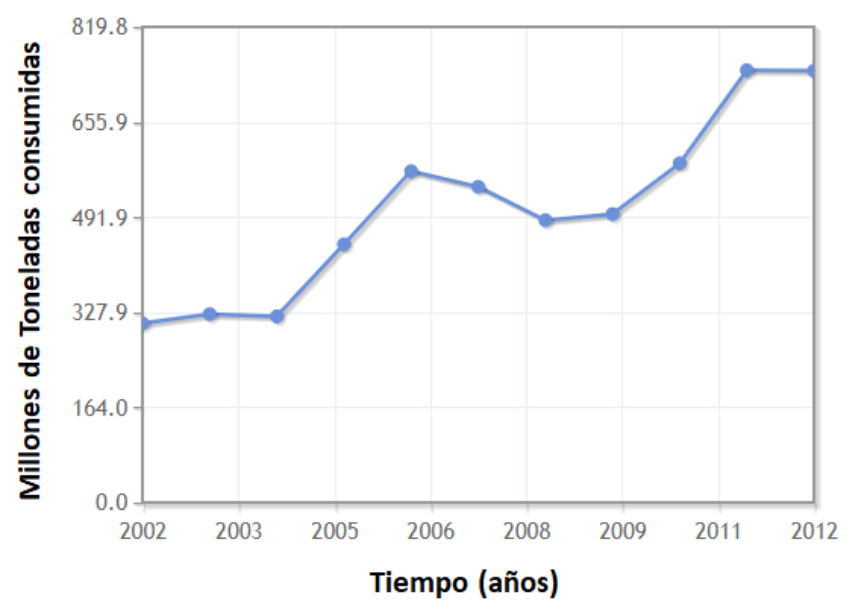

Fig. 2. Consumo de fertilizantes en Colombia 2002-2012. [18]

\section{IMPACTO DE LOS FERTILIZANTES NiTROGENADOS}

\section{A. Efecto de los fertilizantes en el ciclo del Nitrógeno}

Teniendo en cuenta que el ciclo del Nitrógeno, el cual se basa en la interrelación de diversos estados de oxidorreducción de este compuesto (Fig. 3), no es un sistema cerrado [20], es claro que como lo afirman Vitousek et al. [21] las actividades humanas no sólo han incrementado los aportes, sino que también han incrementado el movimiento global de diversas formas nitrogenadas a través del aire y el agua.

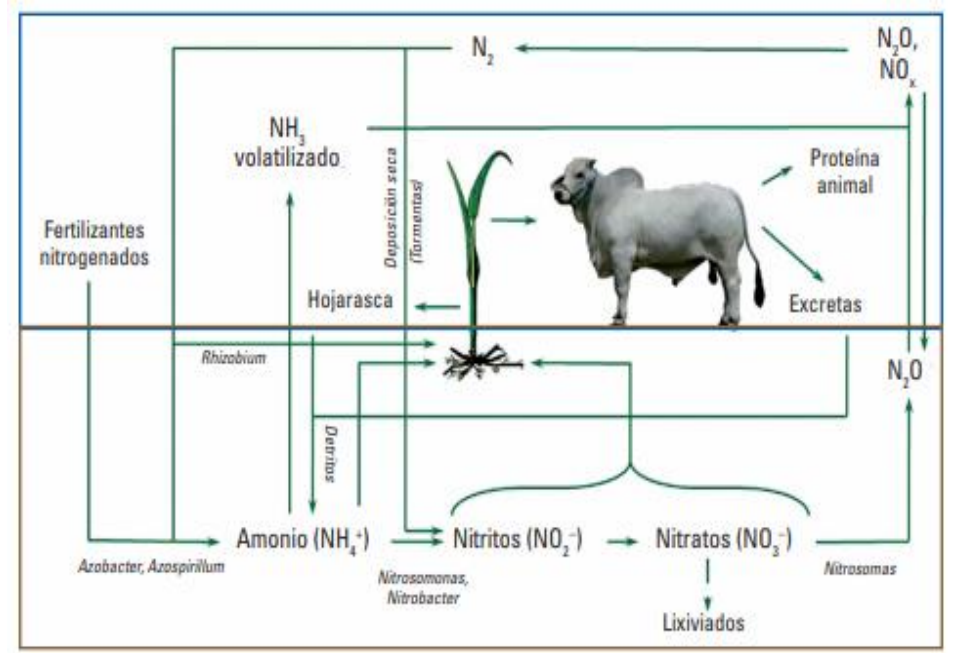

Fig. 3. Esquema representativo del ciclo del Nitrógeno. [22]

Varios son los efectos negativos del uso de estos fertilizantes en el medio ambiente, ya que las raíces de las plantas tan solo pueden absorber dos formas de $\mathrm{N}$, las cuales son nitrato $\left(\mathrm{NO}_{3}{ }^{-}\right)$ principalmente, y amonio $\left(\mathrm{NH}_{4}^{+}\right)$en segundo lugar [19], lo que genera que la cantidad no absorbida quede acumulada al ser inmovilizada por la arcilla, aumentando la acidez y la salinidad en la solución del suelo, lo que a su vez lleva a la perdida de micro y macro nutrientes esenciales, importantes para el desarrollo vegetal y microbiano.

El efecto opuesto se presenta cuando hay un riego excesivo o lluvias constantes, ya que los nitratos son muy solubles, lo que ocasiona que estos sean transportados a las aguas subterráneas volviéndolas más ácidas y ocasionando toxicidad directa para los animales acuáticos [19]. Fugas importantes, se dan cuando se aplican altas concentraciones de amonio y este, por el proceso de nitrificación, se transforma en nitrato, el cual no es adsorbido por el complejo de intercambio catiónico y, por lo tanto, se desplaza hacia abajo con facilidad [21], llegando por escorrentía a los cuerpos de agua y contaminando así otros suelos y fuentes hídricas.

Sepúlveda et al. [16], señalan, por lo tanto, que dichas pérdidas se relacionan fuertemente con el tipo de clima de los ecosistemas, pues en lugares con balances hídricos tendientes a un exceso de agua (precipitación mayor que la evapotranspiración potencial) se favorecen los procesos de lixiviación y por esto el $\mathrm{NO}_{3}{ }^{-}$aportado al suelo y su lixiviación al agua subterránea es un tópico común en el análisis de calidad hídrica.

Además, se afectan los procesos de volatización por la hidrólisis de la úrea que es descompuesta por la enzima ureasa o químicamente hidrolizada a amoniaco $\left(\mathrm{NH}_{3}\right)$ y $\mathrm{CO}_{2}$, gases que son liberados a la atmósfera produciendo el incremento del efecto invernadero y lluvia acida, afectando no solo el suelo y las aguas, si no también el aire y llevando a pérdidas de Nitrógeno que en un inicio había sido aplicado con fines de fertilización [23]; no obstante, es necesario señalar que este efecto de volatilización depende de las condiciones de $\mathrm{pH}$ de la solución de suelo, que regula el equilibrio entre amonio y amoniaco, así como de los contenidos de arcilla, limo y arena para cada suelo [24].

Estos procesos son explicados por Kolenbrander [20], quien señala que el Nitrógeno orgánico debe mineralizarse primero antes de que esté disponible para los cultivos, y dado que la mineralización es un proceso microbiano, parte del Nitrógeno se mineraliza fuera del período de crecimiento activo de la planta en el suelo cultivado. En consecuencia, bajo las condiciones de Europa Occidental, el Nitrógeno está más sujeto a la lixiviación porque se aplica justo antes de que comience el crecimiento activo del cultivo, en un período en que el balance hídrico se vuelve negativo por una evapotranspiración creciente. Cuanto mayor es la entrada de Nitrógeno orgánico, mayor es el riesgo de mayores pérdidas por lixiviación. Sin embargo, desde un punto de vista ambiental, esta pérdida por lixiviación puede reducirse por una tasa creciente de desnitrificación de nitrato. 
Dicha desnitrificación, se realiza en la capa superior del suelo $(1 \mathrm{~m})$, pues es la zona en la que las plantas presentan la mayor proporción de raíces y por ende, la mayor diversidad microbiana rizosférica que participa en este proceso; más allá de esta profundidad la falta de energía reduce la tasa de desnitrificación [20].

Otro contaminante importante generado por el desbalance antrópico causado sobre el ciclo del Nitrógeno es el óxido nitroso $\left(\mathrm{N}_{2} \mathrm{O}\right)$, gas de efecto invernadero que tiene un potencial de calentamiento de 298 veces más que el mismo $\mathrm{CO}_{2}$ y cuyas cantidades se han venido incrementando en la atmósfera. Lo anterior se da debido a que una vez se volatiliza el amoniaco, este puede servir como precursor del $\mathrm{N}_{2} \mathrm{O}$ y el óxido nítrico (NO), compuestos que reaccionan en la estratosfera debilitando la capa de ozono, para caer finalmente al suelo en forma de ácido nítrico $\left(\mathrm{HNO}_{3}\right)$ (componente de la lluvia ácida) [25,26]. Puntualmente en Colombia, las emisiones de $\mathrm{N}_{2} \mathrm{O}$, han venido en aumento, como lo demuestran las cifras reportadas por Nieves \& Olarte [27] quienes señalan que los suelos agropecuarios son la fuente principal de este gas (Fig. 4) a causa del uso intensivo de fertilizante nitrogenado, sin conocer los valores o tiempos de asimilación del mismo en el suelo.

Por otra parte, estudios como el de Li et al. [28] han comprobado que al comparar la capacidad metabólica de los microorganismos presentes en ecosistemas contaminados con fertilizantes y sin contaminar, estos presentan reducción en el pool de genes que les proveen la capacidad de fijar Nitrógeno, mientras que muestran un metabolismo aumentado respecto a la generación de gases de efecto invernadero como el metano o los sulfatos, lo que evidencia cambios en las comunidades y el metabolismo microbiano a causa de este tipo de contaminantes.

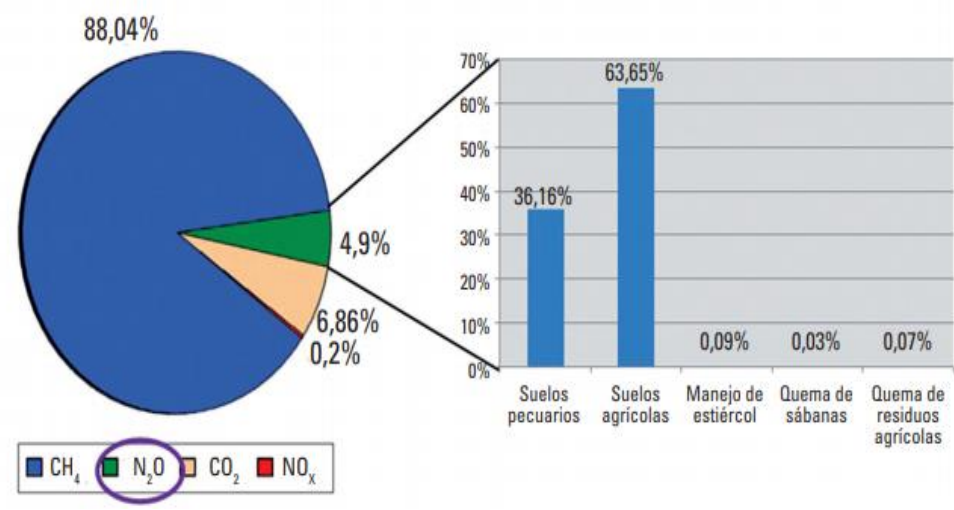

Fig. 4. Emisiones del GEI en el sector agropecuario colombiano en el año de 2004, con énfasis en $\mathrm{N}_{2} \mathrm{O}$. [27]

Teniendo en cuenta que las elevadas dosis de fertilizantes nitrogenados aplicadas a los suelos dan cuenta de insuficientes datos experimentales para calcular las cantidades adecuadas, se han realizado investigaciones como la de Yepis et al. [3] en la que además de determinar el nivel óptimo para el N, se estudió su dinámica aplicada como fertilizante en diferentes dosis comprendidas entre $0-240 \mathrm{~kg} \mathrm{~N} / \mathrm{Ha}$ durante 8 semanas en pequeñas parcelas dedicadas al cultivo por trasplante del tomate variedad Rossol. Este estudio, permitió observar que las formas nítricas priman sobre las amoniacales a medida que transcurre el tiempo, principalmente debido al efecto microbiano en la transformación química de este compuesto, lavándose hacia aguas subterráneas y posterior recirculando en aguas superficiales [16].

Además, se encontró que dosis excesivas (iguales o superiores a $180 \mathrm{~kg} / \mathrm{Ha}$ de $\mathrm{N}$ ), hacen que se genere acumulación de $10 \mathrm{mg}$ de $\mathrm{NO}_{3}-$ por cada $100 \mathrm{~g}$ de suelo, lo que es alarmante, sumado a los contenidos de NO presentes en las aguas de pozos de abasto de la zona, las cuales en el $60 \%$ de los casos, igualaron o superaron el contenido máximo admisible de Nitrógeno, de acuerdo a lo establecido por la OMS para las aguas de consumo humano $[3,16]$.

Cifras similares han sido obtenidas en otros estudios como el de Wagh, et al. [29] quienes al realizar mediciones de nitratos en el agua del río Kadava en India, encontraron que alrededor del $60 \%$ de las muestras no eran aptas para el consumo debido a que excedían los contenidos de nitrato permitidos, lo que se relacionaba con el riesgo de exposición dérmica a estos compuestos al ducharse, siendo los más afectados los niños y los ancianos ya que su piel es más delicada.

Para evitar esto, los investigadores Arias, Pérez \& Rueda [30], indican que se debe establecer o al menos predecir el comportamiento de los contaminantes nitrogenados como el nitrato y otros iones en un sistema agrícola o ecosistema determinado, para lo cual es necesario considerar: la estructura y contenido de materia orgánica de los suelos, sus propiedades mineralógicas, la velocidad de aplicación del flujo de agua y su intensidad, la concentración de nitratos aplicada, el grado de saturación de los nitratos, la densidad aparente, la heterogeneidad de los materiales adsorbentes del suelo y el $\mathrm{pH}$, entre otras.

\section{B. Impacto ambiental y efectos en la salud del uso excesivo de fertilizantes nitrogenados}

Como se ha visto a lo largo del texto, a pesar de su importancia para la seguridad alimentaria y la economía de los países, es clave mencionar respecto a los fertilizantes nitrogenados, que su uso, dosificación y comportamiento químico en suelos y aguas, ha implicado riesgos de contaminación ambiental y en la salud de las poblaciones. Por ejemplo, Peña-Cabriales et al. [2], afirman que a pesar que se emplean fertilizantes nitrogenados de forma constante, este elemento sigue siendo limitante para la producción agrícola, pues se ha comprobado que sólo se absorbe de forma efectiva entre un 10 y un $60 \%$ del producto aplicado. Lo que está relacionado con la alta cantidad de lixiviación del $\mathrm{N}$ no absorbido que llega a aguas subterráneas como nitratos, en donde causa eutroficación $[1,11]$. 
Lo anterior sumado a que su aplicación excesiva conlleva a la degradación de la calidad del suelo por salinidad ("ensalitramiento") [11] y a que se presentan pérdidas por volatilización de amoniaco $\left(\mathrm{NH}_{3}\right)$, lo que tiene un efecto en la salud de los trabajadores agrícolas y las pérdidas por erosión [1]. Además, se observa que la cantidad de $\mathrm{N}$ aplicado es ineficiente desde varios puntos de vista, incluyendo el económico y el ambiental.

Es así como para comprender el impacto de las especies nitrogenadas provenientes de fertilizantes en el ambiente, se hace necesario definir contaminación, entendiéndola como toda causa que contribuya a que un determinado medio o recurso sea inapropiado para su uso alterando su composición, de suerte tal que resulte menos apta para cualquiera o todas las funciones y propósitos para las que sería apropiada en estado natural [31].

En este sentido, Hallberg [32], Merino \& Arozarena [33] y Sagardoy [34], entre otros, plantean que la agricultura que utiliza elevadas aplicaciones de fertilizantes minerales, especialmente los nitrogenados, es la principal responsable del contenido de nitratos en las aguas superficiales y subterráneas, los cuales pueden llegar a ser tóxicos a determinados niveles [35], coincidiendo con Kolenbrander [20] y Vitousel et al. [21] quienes enfatizan en que el aumento de la movilidad, en conjunto con el exceso de Nitrógeno por aportes antropogénicos tiene serias consecuencias ambientales a largo plazo para grandes regiones del planeta.

Así mismo, Vitousel et al. [21] señalan que dentro de los principales impactos, que como consecuencia del uso de fertilizantes nitrogenados por el hombre han afectado el ecosistema, se tienen los siguientes:

- Aumento de las concentraciones globales del gas de efecto invernadero óxido nitroso $\left(\mathrm{N}_{2} \mathrm{O}\right)$.

- Incremento regional de otras formas de óxidos de Nitrógeno (incluyendo óxido nítrico, NO) que conducen a la formación de smog fotoquímico.

- Perdida de Ca y K en los suelos por reacción con especies nitrogenadas, afectando su fertilidad a largo plazo.

- Acidificación substancial de suelos y cuerpos de agua ribereños y lacustres de diversas regiones

- Incrementos excesivos en el transporte de Nitrógeno por los ríos hacia los estuarios y aguas costeras en donde se constituye en un contaminante principal.

- Aceleración de la pérdida de diversidad biológica, especialmente entre plantas adaptadas a suelos pobres en Nitrógeno y subsecuentemente, de los animales y microorganismos que dependen de dichas plantas.

- Cambios en la vida vegetal y animal, así como en los procesos ecológicos estuarinos y costeros, contribuyendo a la disminución a largo plazo de la producción pesquera marina.

Respecto a los efectos en la salud humana, la OMS, manifiesta que las concentraciones de $\mathrm{NO}_{3}{ }^{-}$en fuentes hídricas para consumo humano, no deben superar los $50 \mathrm{mg} / \mathrm{L}$, ya que este compuesto es tóxico una vez se reduce a $\mathrm{NO}_{2}$, pues este último oxida la hemoglobina a metahemoglobina inhabilitándola para transportar oxígeno a los tejidos (metahemoglobinemia), ocasionando cianosis $y$, en concentraciones mayores asfixia, por lo que este tipo de intoxicación a causa de Nitrógeno se conoce como el síndrome de los niños azules. Por su parte, el amoniaco cobra importancia sobre la salud especialmente de los trabajadores agrícolas ya que tras abonar, sus concentraciones pueden superar las 3,000 ppm y entrar al organismo a través de vías respiratorias o dérmicas causando, de acuerdo a la concentración, tiempo de exposición y factores individuales, desde irritación y piquiña, hasta quemaduras o cáncer [36].

Es así que, se puede afirmar que la contaminación por fertilizantes nitrogenados es amplia, y al presentarse de forma directa e indirecta, se hace difícil identificar con exactitud la fuente puntual de dicho impacto, lo que se agudiza al ser en la mayoría de los casos, una contaminación intermitente, dado que está relacionada a actividades estacionales propias de la agricultura, como la época de fertilización o fenómenos ocasionales como la ocurrencia de altas precipitaciones [37].

De ahí que en el presente documento se recalque, el tratar de reducir las dosis de $\mathrm{N}$ aplicado como fertilizante al suelo, sin disminuir los rendimientos, pues ya han sido demostrados los perjuicios que en el transcurso de los años puede continuar ocasionando el uso inadecuado de elevadas cantidades de Nitrógeno aportadas por el abonado mineral.

\section{SOluciones Desde Las Ciencias Básicas Y APLICADAS}

Dentro de las soluciones planteadas, se ha revisado el tratamiento del suelo con inhibidores de la nitrificación, no obstante, esto presenta inconvenientes, ya que los cultivos que reciben solo Nitrógeno amónico no crecen tan bien como los que reciben nitrato [3].

Ante esta situación, se hace necesario contar con tecnologías aplicables a la gestión razonada del Nitrógeno en los sistemas de producción agrícola con el fin de hacerlos sustentables, aportando las cantidades mínimas necesarias para alcanzar el máximo rendimiento potencial de los cultivos.

Por esta razón, en los últimos años se ha venido haciendo énfasis en el uso de microorganismos y plantas para la biorremediación de ambientes contaminados, acompañado de su empleo como biofertlizantes y biopesticidas o controladores biológicos, lo que permite cerrar el ciclo orgánico en procesos productivos agrícolas generando un menor impacto [38].

Para el caso puntual de esta revisión se seleccionaron los microorganismos, dado que presentan una gran diversidad metabólica, lo que les permite un rango amplio de aplicaciones en ambientes y condiciones variadas, dentro de las que se incluyen concentraciones altas de contaminantes. 


\section{A. Biorremediación de suelos impactados con exceso de fertilizantes nitrogenados}

Las principales técnicas de biorremediación consisten en el uso de microorganismos (levaduras, hongos filamentosos o bacterias) para descomponer o degradar sustancias peligrosas en sustancias menos tóxicas [39,40]. Ciertos microorganismos, especialmente las bacterias, poseen genes que codifican para enzimas capaces de emplear sustancias orgánicas peligrosas para los seres humanos, como por ejemplo, combustibles, desechos orgánicos, residuos agrícolas, solventes o sustancias peligrosas, y descomponerlos a productos inocuos, principalmente gases y agua. Además, cuando ya el contaminante ha bajado su concentración y ha sido degradado, las poblaciones de microorganismos empleados para biorremediar, se reducen pues se ha agotado su fuente de alimento, sin que los microorganismos muertos presenten riesgos de contaminación [41,42].

Las medidas de biorremediación pueden usarse como método para descontaminar tanto suelos como aguas, clasificándose en dos grandes categorías: «in situ» y «ex situ». Las estrategias «in situ» tratan el suelo o agua contaminado en el lugar de origen. Por el contrario, las medidas «ex situ» consisten en excavar, trasportar y tratar la matriz contaminada fuera de la zona de procedencia [43-45].

Teniendo en cuenta que el ciclo del Nitrógeno se basa en la interrelación de diversos estados de oxidorreducción de este compuesto (Fig. 3), y a que se ha comprobado que dentro de las especies de $\mathrm{N}$, el más móvil y el que se asimila más fácilmente es el $\mathrm{NO}_{3}^{-}[19,46]$, se ha planteado la posibilidad de adicionar a los suelos y aguas, microorganismos externos, principalmente de tipo comercial (bioaumentación) que sean capaces de realizar la reducción de nitrato a $\mathrm{N}_{2}$ y de $\mathrm{NH}_{4}{ }^{+}$a $\mathrm{NH}_{3}{ }^{+}$para así por medio de la desnitrificación (Fig. 5), disminuir las concentraciones de este compuesto en el suelo evitando la contaminación [18].

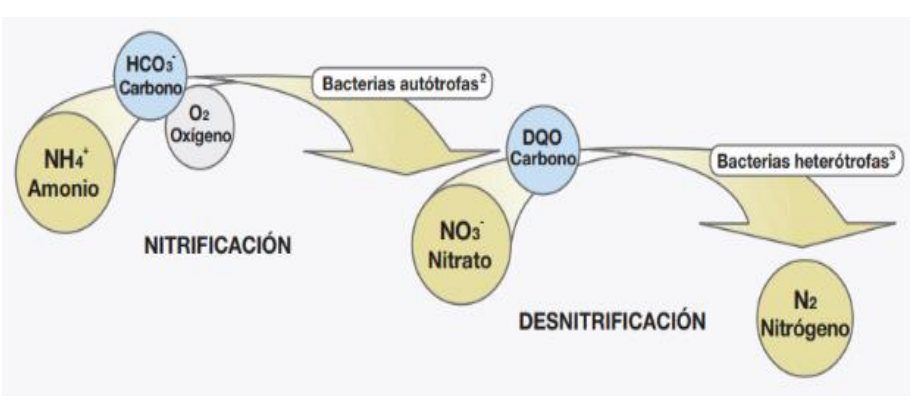

Fig. 5. Reacción de oxidación (nitrificación) y de reducción (desnitrificación) que tienen lugar en el proceso de nitrificacióndesnitrificación (NDN). [45]

En ocasiones no se requiere adicionar microorganismos exógenos para realizar dicha descontaminación, pues se emplea la estrategia denominada bioestimulación, en la cual, por medio de la adición de nutrientes que favorezcan el crecimiento de los microorganismos propios del suelo contaminado, se consigue remover el $\mathrm{N}$ a través de desnitrificación y por tanto, reducir el potencial de contaminación. Lo anterior, se logra estimulando a las bacterias heterótrofas anaerobias que usan nitratos como aceptor final de electrones en sus procesos respiratorios hasta llegar a la producción de gas $\mathrm{N}_{2}$, pasando por intermediarios como $\mathrm{NO}_{2}$, óxido nítrico (NO) y óxido nitroso $\left(\mathrm{N}_{2} \mathrm{O}\right)$; aunque también los procesos de desnitrificación pueden darse en aerobiosis $[16,48]$.

Las bacterias capaces de reducir nitratos están ampliamente distribuidas en ecosistemas acuáticos y terrestres y son filogenéticamente diversas. Su función ha sido demostrada en géneros como Bacillus, Pseudomonas, Acinetobacter, Micrococcus, Achromobacter, Bradyrhizobium, Clostridium, Corinebacterium y Actinomyces, entre otros, reduciendo los niveles de nitratos tanto de forma individual como en consorcios microbianos establecidos [16,48]. De hecho, Benavides et al. [48] afirman que al estar en consorcio las bacterias desnitrificantes, pueden combinar y complementar sus funciones metabólicas y de forma colectiva, desnitrificar el suelo a mayores tasas, lo que a su vez se complementa con el hecho de que, al formar parte del consorcio, las diferentes especies bacterianas pueden tolerar los cambios físico-químicos que se den en el ambiente durante el proceso de biorremediación.

Otra de las estrategias ampliamente utilizadas para la remoción de compuestos nitrogenados, especialmente en agua, es el uso de microalgas dentro de las que se destacan los géneros Chlorella, Spirullina y Chlamydomonas. Estas no sólo tienen efecto sobre los compuestos nitrogenados, sino también sobre el Fósforo, pesticidas, herbicidas y metales pesados, entre otros compuestos, debido a su capacidad para acumular importantes concentraciones de compuestos tóxicos, sin afectar su actividad biológica, al mismo tiempo que liberan $\mathrm{O}_{2}$ por medio de la fotosíntesis, mejorando la aireación en los cuerpos de agua $[48,49]$.

Dentro de los géneros de microalgas, los estudios coinciden en que la remoción de nitratos, nitritos y fosfatos en aguas contaminadas es mayor con Chlorella, con porcentajes de remoción mayores al 50\% durante el primer día [49], alcanzando valores máximos del $88.24 \%$, para nitratos y $87.2 \%$, para nitritos en el cuarto día del tratamiento [50].

Ortiz-Villota et al. [50] afirman que el éxito de la biorremediación de compuestos nitrogenados en suelos y aguas con bacterias o microalgas, está dado por la capacidad que tienen estos microorganismos de emplear este elemento como parte de su metabolismo, para sintetizar aminoácidos, proteínas y ácidos nucleicos necesarios para la construcción del protoplasma.

Numerosos estudios han demostrado que cada especie, e incluso cada cepa tiene requerimientos nutricionales diferentes y que al variar las fuentes de Nitrógeno en el medio se afecta en 
mayor o menor medida el crecimiento microbiano [50], dependiendo de si lo que se emplea son fuentes de Nitrógeno complejas como las encontradas en aguas residuales (suero de leche, la harina de maíz y la cáscara de papa) o sencillas como lo son directamente los compuestos nitrogenados encontrados en los fertilizantes; en la Fig. 6 se presenta un ejemplo de dicho efecto. Así mismo, los cambios en el crecimiento microbiano en respuesta a diversas fuentes de Nitrógeno, están dados e involucran directamente cambios en la actividad enzimática observada, ya que las enzimas activas en cada caso varían.

Lo anterior, sugiere el uso de microorganismos como una estrategia viable para la recuperación de suelos y aguas impactadas con fertilizantes nitrogenados, siempre y cuando se establezcan de forma adecuada los parámetros que permitan su propagación en dichos ambientes

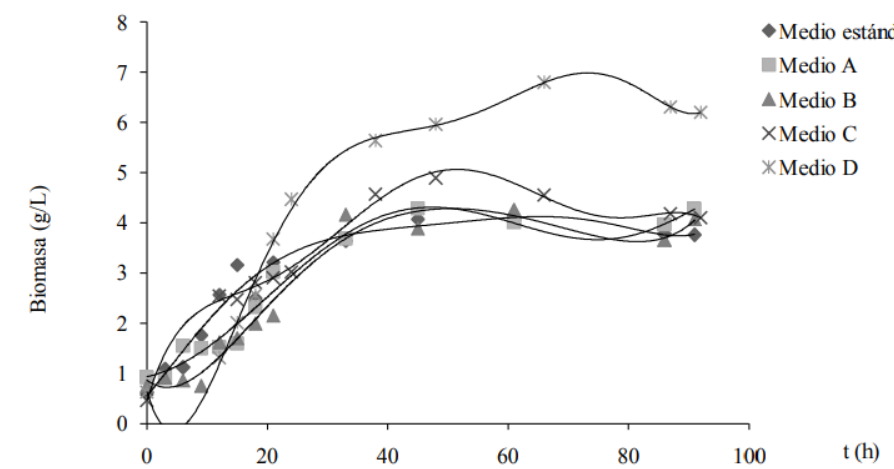

Fig. 6. Efecto del reemplazo de la fuente de Nitrógeno sobre el crecimiento de un microorganismo. [49]

\section{B. Uso de microorganismos como estrategia de fertilización sostenible.}

Los denominados biofertilizantes pueden ser descritos como productos que contienen células viables de diversos tipos de microorganismos, los cuales, al ser aplicados a las semillas, la superficie de las plantas o los suelos, colonizan la rizósfera o el interior de las plantas y promueven su crecimiento al convertir elementos nutricionalmente importantes (Nitrógeno, Fósforo) de fuentes no disponibles, a fuentes biológicamente disponibles a través de procesos enzimáticos [52].

Los microorganismos asociados a las raíces de las plantas mejoran, estimulan y facilitan el sano desarrollo de la planta a dosis inferiores de fertilizantes nitrogenados, fosforados u otros necesarios para un rendimiento rentable. Estos microorganismos son mayoritariamente de naturaleza bacteriana y mediante su correcta aplicación permiten, en el caso del Nitrógeno, fijar biológicamente $\mathrm{N}_{2}$, lo que implica menores importes de Nitrógeno al cultivo, al mismo tiempo que mejora la absorción de fertilizantes, además de proteger a las raíces del ataque de fitopatógenos [53].

Las bacterias fijadoras de Nitrógeno se pueden clasificar en dos grandes grupos: 1) Aquellas que realizan la fijación en estrecha asociación con las raíces de las plantas y su simbiosis es obligada e incluso especie dependiente; grupo en el que se encuentra el género Rizobium y su asociación con las leguminosas, las cianobacterias asociadas a plantas de tipo arbustivo y a hongos, y Frankia, género que se asocia especialmente con árboles; 2) Aquellas que se consideran de vida libre al estar presentes en la rizosfera de la planta, pero no presentar simbiosis obligada; este grupo está constituido especialmente por los aerobios Azotobacter y Bejeirinkia, microaerofílicos como Azospirillum y anaerobios como Clostridium y Desulfovibrio. En todos los casos, estos microorganismos cumplen esta función debido a que presentan el sistema enzimático denominado nitrogenasa que les permite reducir el Nitrógeno atmosférico $\left(\mathrm{N}_{2}\right)$ a amonio [54].

Para fines prácticos respecto al uso de microorganismos como fijadores de Nitrógeno que permitan disminuir las cantidades de este nutriente aplicados a través de fertilizantes sintéticos a los cultivos, se plantea que las bacterias a emplear sean de vida libre, pues se ha comprobado que su espectro de acción es amplio en diversos suelos y para un variado número de plantas, siendo estas rizobacterias beneficiosas para el crecimiento vegetal, al ser promotoras del crecimiento de las plantas (PGPR por sus siglas en inglés), mediante la colonización de las raíces (rizósfera) en donde ayudan a generar un importante entorno ecológico para las interacciones plantamicroorganismo [52].

Como se mencionó, dentro de este grupo se encuentran los microorganismos simbióticos que incluyen las cianobacterias fijadoras de Nitrógeno de vida libre y bacterias pertenecientes a los géneros Azotobacter, Azospirillum, Anabaena, Enterobacter, Klebsiella, Pseudomonas, entre muchas otras, para las cuales se ha demostrado que se adhieren a la raíz y colonizan de manera eficiente las superficies de la misma generando beneficios para el microorganismo y la planta asociados [52].

Bhat et al. [52], señalan que además del beneficio obvio respecto a la nutrición vegetal al suplir los requerimientos de Nitrógeno, varias asociaciones entre las plantas y las fijadoras muestran una respuesta protectora en condiciones ambientales restrictivas, como el estrés salino. Dicho efecto favorable puede atribuirse directamente a las bacterias o indirectamente, al efecto sobre la fisiología de la planta, ya que estos microorganismos producen metabolitos como los polisacáridos que pueden modificar la estructura del suelo y tienen un efecto positivo en las plantas que crecen en el estrés hídrico. Además, las bacterias también pueden estimular a la planta a activar una actividad metabólica particular como, por ejemplo, aumentar sus exudados y, en consecuencia, mejorar las cualidades del suelo rizosférico protegiendo a las plantas contra la colonización patógena.

El uso de bacterias fijadoras de Nitrógeno ha sido reportado para numerosos cultivos dentro de los que se resaltan la soya, el fríjol, el arroz, la caña, el trigo y el maíz, a partir de los cuales 
se han aislado además, micoorganismos para su posterior propagación y uso comercial [55]. Es así como respecto a la fijación de Nitrógeno se continúan realizando estudios en los que se evalúan especialmente, sus efectos en cultivos de consumo masivo como la soya y el fríjol para lo cual se han empleado actinomicetes endofíticos como cepas de Bradyrhizobium spp. y Streptomyces griseoflavus con resultados prometedores [56].

De los cultivos anteriormente mencionados, tal vez uno de los más estudiados ha sido el del arroz, para el cual se ha realizado inoculación con bacterias de los géneros Herbospirillum y Burkholderia [57] y otros autores como Mishra \& Pabbli [57] han planteado además, el uso de cianobacterias como una alternativa económica y ecológicamente atractiva para la fertilización de este cultivo, teniendo en cuenta que al realizarse bajo esquemas inundables, el lavado de Nitrógeno puede ser mayor que en otros sistemas, en el que además de fijar $\mathrm{N}_{2}$ y emplear amonio como fuente de $\mathrm{N}$, estos microorganismos favorecerían la aireación al realizar de forma simultánea fotosíntesis.

Estas investigaciones muestran que el uso de bacterias fijadoras de Nitrógeno, especialmente de vida libre y aquellas capaces de realizar simbiosis obligada con las plantas, solas o en conjunto con otros microorganismos PGPR, tienen el potencial de contribuir a la promoción del crecimiento sostenible de las plantas [52], ayudando de esta forma a disminuir el impacto ambiental y en la salud de las personas de los fertilizantes nitrogenados de síntesis química. Esto permite afirmar que los fertilizantes biológicos ayudan a la productividad y la sostenibilidad protegiendo el medio ambiente al mismo tiempo que son más rentables para los agricultores; lo anterior, siempre teniendo en cuenta que el éxito de su uso depende de múltiples factores como la cepa o cepas empleadas, el tipo de cultivo, las características del suelo y condiciones ambientales, entre otras por lo que se deben realizar experimentos en condiciones de campo [59].

Es importante enfatizar en que a pesar de los inegables beneficios económicos y ecológicos de las alternativas planteadas en este documento, tanto para el caso de las cepas empleadas en biorremediación de ambientes contaminados con fertilizantes nitrogenados, como para aquellas a ser aplicadas como biofertilizantes, se deben realizar estudios cuidadosos que permitan determinar que estos microorganismos son seguros para su aplicación y que no tendrán efectos negativos ni en el ambiente ni en la salud de humanos o animales.

\section{CONCLUSIONES}

Como se evidencia, la contaminación y degradación de los ecosistemas es la problemática emergente de mayor preocupación del siglo XXI, pues los daños ambientales repercuten en las demás esferas de la sociedad, por lo que estudios en los que se den soluciones apoyadas en el desarrollo sustentable se hacen cada vez más necesarias.
Este es el caso de los productos a base de microorganismos para los cuales se ha demostrado de forma exitosa y ampliamente documentada que funcionan, tanto en procesos de biorremediación de ambientes contaminados, como en su uso como biofertilizantes y promotores de crecimiento vegetal, brindando soluciones efectivas que permiten disminuir de cierta medida la dependencia de productos químicos por el sector agrícola, tendencia que de acuerdo a varios autores, se presenta en países industrializados, en los que se ha hecho evidente que para garantizar la sostenibilidad y productividad de los suelos no se recomiendan esquemas de manejo exclusivos con agroquímicos.

Tras la revisión de literatura realizada, es possible afirmar que las condiciones del sector agropecuario tanto a nivel mundial como nacional, requieren del uso más eficiente de los nutrientes para no generar efectos adversos en los ecosistemas, debido a que para continuar siendo productivos deben garantizar la preservación en el tiempo de los recursos suelo, agua y atmósfera.

Por lo anterior, es posible concluir que el durante los últimos 20 años se ha comprobado que uso de microorganismos capaces de utilizar las fuentes de Nitrógeno como estrategia de biorremediación en matrices impactadas con excesos de este nutriente, ha mostrado ser una aproximación eficiente para la remoción de este elemento en suelos y aguas, que da valor agregado a los sistemas agrícolas y sus productos finales, al estar en sintonía con los requerimientos actuales de los mercados en relación a minimizar los impactos ambientales negativos derivados de las actividades productivas agrícolas. Por su parte, el uso de microorganismos fijadores de nitrógeno como biofertilizantes, de forma paralela y complementaria, permite disminuir la aplicación de fuentes nitrogenadas sintéticas, evitando así mismo el lavado de nitratos hacia fuentes de agua y otros suelos previniendo su contaminación.

\section{REFERENCIAS}

[1] F. O. García," El ciclo del nitrógeno en sistemas agrícolas", Jun, 17, 2019. [Online]. Disponible en http://www.econoagro.com/images/stories/pdf/agricultura/CicloNit rogeno.pdf

[2] J. J. Peña-Cabriales, O. A. Grageda-Cabrera, J. A. Vera-Nuñez, "Manejo de los fertilizantes nitrogenados en México: uso de las técnicas isotópicas $(15 \mathrm{~N})$ ", Terra Latinoamericana, Vol. 20, no. 1, pp. 51-56, Mar. 2002

[3] O. Yepis, O. Fundora, C. Perira, T. Crespo," La contaminación ambiental por el uso excesivo de fertilizantes nitrogenados en el cultivo de tomate", SCIENTIA gerundensis, Vol. 24, pp. 5-12, 1999.

[4] S. Navarro, G. Navarro, Química Agrícola, Madrid, España, 2000, pp. 487

[5] H. A. García-Perdomo, "Conceptos fundamentales de las revisiones sistemáticas/metaanálisis", Urología colombiana, Vol.24, no. 1, pp. 28-34, Abr. 2015. DOI: 10.1016/j.uroco.2015.03.005

[6] B. Moreno, M. Muñoz, J. Cuellar, S. Domancic, J. Villanueva, "Revisiones Sistemáticas: definición y nociones básicas", Revista clínica de periodoncia, implantología y rehabilitación oral, Vol. 11, no. 3, 2018. DOI: 10.4067/S0719-01072018000300184 
[7] A. Julca-Otiniano, L. Meneses-Florián, R. Blas-Sevillano, S. BelloAmez, "La Materia Orgánica, Importancia Y Experiencia De Su Uso En La Agricultura", Idesia (Arica), Vol. 24, no. 1, pp. 49-61. Abr. 2006, DOI: 10.4067/S0718-34292006000100009

[8] Organización de las Naciones Unidas para la Alimentación y la Agricultura (FAO). Enseñanzas de la revolución verde: hacia una nueva revolución verde. Presentado en Cumbre mundial sobre la alimentación, Roma Italia, Nov. 13-17, 1996 [Online]. Disponible en http://www.fao.org/3/w2612s/w2612s06.htm

[9] R. Tapia, "La revolución verde y plaguicidas en el ambiente la granja", Revista de ciencias de la vida, Vol. 4, pp. 47-50. 2006, DOI: 10.17163/lgr.n4.2005.06

[10] G. Navarro, Química Agrícola, México D.F, México, 2011, pp. 319.

[11] R. Cárdenas-Navarro, J. M. Sánchez-Yánez, R. Farías-Rodríguez, J. J. Peña-Cabriales, "Los aportes del nitrógeno a la agricultura", Revista Chapingo Serie Horticultura, Vol. 10, no. 2, pp. 173-178. 2004, DOI: 10.5154/r.rchsh.2002.07.039

[12] L. Taiz, A. Kirkby, Plan physiology, Washingtong D.C, USA, 1998.

[13] K. Mengel, A. Kirkby, Principles of Plant Nutrition. Netherlands, 2001. pp. 210

[14] Agrohuerto, "Nutrientes que necesitan las plantas. Algunos trucos para detectar su carencia. Guía visual de síntomas". May, 10, 2019. [Online]. Disponible https://agroalimentando.com/nota.php?id_nota $=8232$

[15] P. Parodi, Los desafíos de la agronomía. Temulco, Chile, 2010.

[16] A. Sepúlveda, E. González, C. Inostroza, "Remediación de la contaminación por nitratos en el suelo: Antecedentes generales y pertinencia en zona sur de Chile", Gestión Ambiental, Vol. 21, pp. 13-32, 2011. Disponible en http://repositoriodigital.uctemuco.cl/bitstream/handle/10925/531/G A_21_Sepulveda\%282011\%29.pdf?sequence=1

[17] FERTINAL. "Fertilizantes disponibles en México", Jun, 01, 2019. [Online]. Disponible en https://www.grupofertinal.com.mx/

[18] Indexmundi, "Colombia, consumo de fertilizantes", May, 25, 2019. [Online]. Disponible https://www.indexmundi.com/es/datos/colombia/consumo-defertilizantes

[19] J. M. Durán, N. Retamal, R. Montiel, "El papel del nitrógeno en la agricultura y la contaminación por nitrato". Departamento de Producción Vegetal (Fitotecnia) de la ETSIA (Universidad Politécnica de Madrid). Disponible en http://www.interempresas.net/Agricola/Articulos/39819-El-papeldel-nitrogeno-en-la-agricultura-y-la-contaminacion-pornitrato.html

[20] G. J. Kolenbrander, "Nitrogen in organic matter and fertilizer as a source of pollution", Conference on Nitrogen As a Water Pollutant, Vol. 8.4, pp. 67-84. 2013, DOI: 10.1016/B978-1-4832-13446.50006-X

[21] P. M. Vitousek, J. A. Chair, R. W. Howarth, G. E. Likens, P. A Matson, D. Schindler, W. H. Schlesinger, G. D. Tilman, "Human alteration of the global nitrogen cycle: Sources and consequences", Ecological Applications, Vol. 1, pp. 1-17, 1997. DOI: 10.1890/1051-0761(1997)007[0737:HAOTGN]2.0.CO;2

[22] J. E. Garzón, E. A. Cárdenas, "Emisiones antropogénicas de amoniaco, nitratos y óxido nitroso: compuestos nitrogenados que afectan el medio ambiente en el sector agropecuario colombiano". Rev. Med. Vet. Zoot. Vol. 60. No. II, pp. 121-138. 2013. DOI: $10.15446 / \mathrm{rfmvz}$

[23] F. Ramos Goucy, J. A. Aguilar Rubalcava, C. López Luévano, Lixiviación de nitratos en agricultura Intagri, Jun, 10, 2019. [Online]. Disponible http://www.fiestaspoyiyos.com.mx/Lws/32499/lexiviacion-encultivos.html

[24] I. Ginés, I. Mariscal-Sancho, Incidencia de los fertilizantes sobre el pH del suelo. Fertiberia S.A. May. 30, 2019. [Online]. Disponible en http://oa.upm.es/3176/2/MARISCAL_MONO_2002_01.pdf

[25] E. Sanhueza, "The role of the atmosphere in nitrogen cycling", Plant Soil, Vol. 67, pp. 61-71, 1982.

[26] E. A. Cárdenas," Evaluación de una alternativa para disminuir el impacto que causan los fertilizantes nitrogenados en las pasturas de clima frío en Colombia", Tesis de maestría en ciencias agrícolas, Fac. Agron., Universidad Nacional de Colombia., Bogotá, 2003.

[27] H. E. Nieves, C. Olarte, "Resumen técnico del módulo de agricultura del inventario nacional de GEIS, años 2000 y 2004".
Bogotá (Colombia): Instituto de Hidrología, Meteorología y Estudios Ambientales (IDEAM), pp. 3, 8-9.

[28] Y. Li, L. Zheng, Y. Zhang, H. Liu, H., "Comparative metagenomics study reveals pollution induced changes of microbial genes in mangrove sediments" Scientific Reports.Vol. 9, no. 1. 2019. DOI. 10.1038/s41598-019-42260-4

[29] V. M. Wagh, D. B. Panaskar, S. V. Mukate, M. L. Aamalawar, U. Laxman Sahu, U. "Nitrate associated health risks from groundwater of Kadava River Basin Nashik, Maharashtra, India", Human and Ecological Risk Assessment: an international journal, Vol. 26, no 3, 2020. DOI: 10.1080/10807039.2018.1528861

[30] S. Arias, J. Pérez, O. Rueda. "Lixiviación de nitratos en dos suelos al alterar sus propiedades físicas", Revista EIA, Vol. 2, pp. 35 - 40, 2004. DOI: 10.24050/reia.v1i2.130

[31] C. Ablenda, D. González, A. López, J. Lungo, T. Nuñez, E. Pérez. "El agua subterránea es más que $\mathrm{H}_{2} \mathrm{O}$ " Revista Tierra Amiga. pp. 29-36, 1996

[32] G.R. Hallberg, "Nitrate in groundwater in the United States". En: Nitrogen manegement and groundwater protection. R.F. Follet, Ed. Amsterdam: Elsevier, 1989

[33] D. Merino, J. Arozarena. "Nitratos en hortalizas", Agrícola Vergel, Vol X, no. 120, pp. 25-27, 1991.

[34] J. A. Sagardoy, Una visión global de la contaminación del agua por la agricultura. Presentado en Prevención de la contaminación del agua por la agricultura y actividades afines. Informe sobre temas hídricos. FAO. Roma. Italia. pp. 50-5232.

[35] P. Domínguez, A. Dominguez, "Nitratos en hortalizas españolas". Agrícola Vergel. Vol. XII, no. 147, pp. 18-20, 1994

[36] Agency for Toxic Substances and Disease Registry (ATSDR), "Resumen de salud pública, Amoniaco", Jun, 15, 2019. [Online]. Disponible en https://www.atsdr.cdc.gov/es/phs/es_phs126.pdf

[37] S. Carpenter, N. Caraco, D. Correll, R. Howarth, A. Sharpley, V. Smith "Nonpoint pollution of surface waters with phosphorous and nitrogen". Ecol. Soc. Am. Issues in Ecology, no. 3, 1998. DOI: 10.1890/1051-0761(1998)008[0559:NPOSWW]2.0.CO;2

[38] M. Ahmad, L. Pataczek, T. H. Hilger, Z. A. Zahir, A. Hussain, F. Rasche, R. Schafleitner, S. Ø. Solberg, "Perspectives of Microbial Inoculation for Sustainable Development and Environmental Management" Frontiers in microbiology. Vol. 9. Article 2992, 2019. DOI: $10.3389 / \mathrm{fmicb} .2018 .02992$

[39] R. Boyer. Conceptos de Bioquímica. México, D.F, México, 2000.

[40] F. Bento, F. Camargo, B. Okeke, W. Frankenberger, "Comparative bioremediation of soils contaminated with diesel oil by natural attenuation, biostimulation and bioaugmentation", Bioresource Technology. Vol. 96, pp. DOI: 1049-1055, 2005. /10.1016/j.biortech.2004.09.008

[41] M. Leung, "Bioremediation: Techniques for cleaning up a mess" BioTeach Journal, Vol. 2, pp. 18-22, 2004. DOI: 10.12691/ijebb-31-5

[42] K. Nealson. "Harnessing microbial appetites for remediation", Nature Biotechnology, Vol. 21, pp. 243- 245, 2003. DOI: $10.1038 / \mathrm{nbt} 0303-243$

[43] P. M. Hatzinger, M. Whittier, M. Arkins, C. Bryan, W. Guarini. "InSitu and ExSitu Bioremediation Options for Treating Perchlorate in Groundwater" Remediation Journal, Vol. 12, no. 2, pp. 69 - 86, 2002. DOI: $10.1002 / \mathrm{rem} .10026$

[44] L. Xul, H, Lee. "Novel approach to microwave eassisted extraction and micro-solid-phase extraction from soil using graphite ûbers as sorbent", Journal of Chromatography, Vol. 1192, pp. 203-207, 2008. DOI: 10.1016/j.chroma.2008.03.060

[45] W. Chen, L. Hou, X. Luo, L. Zhu. "Effects of chemical oxidation on sorption and desorption of PAHs in typical Chinese soils", Environmental Pollution, Vol. 157, no. 1894-1903, 2009. DOI: /10.1016/j.envpol.2009.01.027

[46] F. Ramos. "Dinámica del ciclo de nitrógeno en fertilización de maíz", Jun. 03, 2019. [Online]. Disponible en https://www.hortalizas.com/cultivos/dinamica-del-ciclo-denitrogeno-en-fertilizacion-de-maiz/

[47] Arc-cat.net. "Guía de tratamientos de las deyecciones ganaderas, Nitrificación-desnitrificación", May, 17, 2019. [Online]. Disponible en http://www.arccat.net/es/altres/purins/guia/pdf/ficha5.pdf

[48] J. L. Benavidez, G. M. Quintero, O. L. Ostos, "Aislamiento e identificación de diez cepas bacterianas desnitrificantes a partir de un suelo agrícola contaminado con abonos nitrogenados proveniente 
de una finca productora de cebolla en la Laguna de Tota, Boyacá, Colombia" Nova. Vol. 4. no. 6, pp. 50-54, 2006. DOI: 10.22490/24629448.360.

[49] J. G. L. Avila Peltroche, A. Laos Ayala, R.M. Verano Silvera, H. Montoya Terreros, "Remoción de nitratos y fosfatos por cepas nativas de Chlorella sp. (Chlorellaceae) y Chlamydomonas $s p$. (Chlamydomonadaceae) libres e inmovilizadas en aguas residuales municipales", Arnaldoa, Vol. 25, no. 2, 2018. DOI: /10.22497/arnaldoa.252.25210

[50] M. T. Ortiz-Villota, M.A. Romero-Morales, L.D. Meza-Rodríguez, "La biorremediación con microalgas (Spirulina máxima, Spirulina platensis y Chlorella vulgaris) como alternativa para tratar la eutrofización de la laguna de Ubaque, Colombia." Rev. investig. desarro. innov, Vol. 9, no. 1, pp. 163-176. 2018, DOI: 10.19053/20278306.v9.n1.2018.8153.

[51] A. Osorio, N. Gómez, C. Sánchez, "Evaluación de diferentes fuentes de carbono y de nitrógeno para la producción de renina a partir del moho Mucor miehei" Rev. Fac. Ing. Univ. Antioquia, Vol. 45, pp, 17-26, 2008.

[52] T. A. Bhat, L. Ahmad, M. A. Ganai, UI-Hag, Shams, O. A. Khan, "Nitrogen Fixing Biofertilizers; Mechanism and Growth Promotion: A Review", Journal Of Pure And Applied Microbiology, Vol. 9. no. 2, pp. 1675-1690, 2015.

[53] J. K. Vessey, "Plant growth promoting rhizobacteria as biofertilizers", Plant Soil. Vol. 255, no. 571-586, 2003. DOI: /10.1023/A:1026037216893

[54] J. Talukdar, M. C. Kalita, B. C. Goswami, "Influence Of Dissolved Inorganic Carbon And Nitrogen Sources On Growth, Total Lipid Content And Calorific Value Of The Freshwater Oleaginous Microalgae Ankistrodesmus Falcatus (Corda) Ralfs", Environmental Research, Engineering and Management, Vol. 3, no. 61, pp. 14-25, 2012. DOI: /10.5755/j01.erem.61.3.1423

[55] R. B. Bhattacharjee, A. Singh, S.N. Mukhopadhyay, "Use of nitrogen-fixing bacteria as biofertiliser for non-legumes: prospects and challenges", Applied microbiology and biotechnology, Vol. 80, no. 2, pp. 199-209, 2008. DOI: 10.1007/s00253-008-1567-2

[56] A. Z. Htwe, S. M. Moh, K. M. Soe, K. Moe, T. Yamakawa, "Effects of Biofertilizer Produced from Bradyrhizobium and Streptomyces griseoflavus on Plant Growth, Nodulation, Nitrogen Fixation, Nutrient Uptake, and Seed Yield of Mung Bean, Cowpea, and Soybean", Agronomy, Vol. 9. no. 2, pp. 77, 2019, DOI: 10.3390/agronomy 9020077

[57] V. L. D. Baldani, J. I, Döbereiner, "Inoculation of rice plants with the endophytic diazotrophs Herbaspirillum seropedicae and Burkholderia spp", Biol Fert Soils, Vol. 30, pp. 485-491, 2000. DOI: $10.1007 / \mathrm{s} 003740050027$

[58] U. Mihra, S. Pabbi, "Cyanobacteria: A potencial biofertilizer for rise", Resonance, Vol. 9, no. 6, pp. 6-10, 2014. DOI: 10.1007/BF02839213

[59] L. E. Fuentes-Ramírez, J. Caballero-Mellado, "Bacterial biofertilizers". En: Biocontol and biofertilization, Z. A. Siddiqui, Ed, Springer. Netherlands. pp. 143-172, 2005.

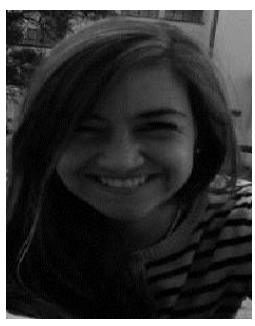

Luisa Alejandra García Galindo. Bióloga y Microbióloga Industrial de la Pontificia Universidad Javeriana, Bogotá, Colombia; Magister en Ciencias de la Universidad Nacional de Colombia, sede Bogotá; candidata a Doctora en Biotecnología de esta misma Universidad. Se ha desempeñado en la planeación, formulación, implementación, comunicación, gestión, control y seguimiento de proyectos de investigación y desarrollo con énfasis en biotecnología, biorremediación ambiental, agroecología, sostenibilidad y biodiversidad en empresas privadas y públicas, en conjunto con instituciones de educación superior, como estrategia de apropiación social del conocimiento. Es reconocida ante el Ministerio de Ciencia, Tecnología e innovación (Minciencias) como investigador Junior. Actualmente se desempeña como docente e investigadora y se encuentra coordinando el Área de Investigaciones de la Facultad de Ciencias Básicas de la Fundación Universitaria Horizonte.

ORCID: https://orcid.org/0000-0001-7785-466X

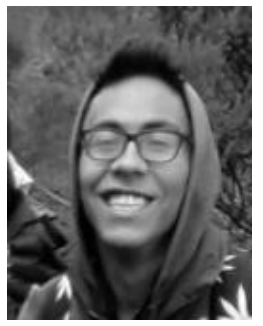

Arzaled Capera Rivas. Estudiante del técnico profesional en Procesos ambientales de la Fundación Universitaria Horizonte, Bogotá, Colombia desde el enero del 2018. Se vinculó al Semillero de Estudios Ambientales (SEAmbiental), a partir de junio de 2018 debido a su interés en profundizar en la solución de impactos ambientales generados por los patrones de consumo de la sociedad contemporánea. Actualmente, se encuentra a la espera de su grado como Técnico para continuar con sus estudios profesionales. ORCID:https://orcid.org/0000-0001-7951-2209

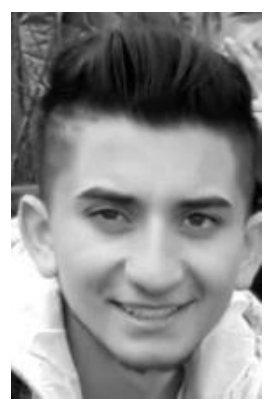

Jean Paul Melendez. Estudiante del técnico profesional en Procesos ambientales de la Fundación Universitaria Horizonte, Bogotá, Colombia desde el enero del 2018. Se vinculó al Semillero de Estudios Ambientales (SEAmbiental), a partir de junio de 2018 debido a su interés en profundizar en la solución de impactos ambientales generados por los patrones de consumo de la sociedad contemporánea. Actualmente, se encuentra a la espera de su grado como Técnico para continuar con sus estudios profesionales.

ORCID: https://orcid.org/0000-0002-7612-9965

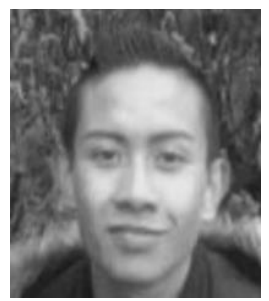

Nicolás Mayorquín. Estudiante del técnico profesional en Procesos ambientales de la Fundación Universitaria Horizonte, Bogotá, Colombia desde el enero del 2018. Se vinculó al Semillero de Estudios Ambientales (SEAmbiental), a partir de junio de 2018 debido a su interés en profundizar en la solución de impactos ambientales generados por los patrones de consumo de la sociedad contemporánea. Actualmente, se encuentra a la espera de su grado como Técnico para continuar con sus estudios profesionales. ORCID: https://orcid.org/0000-0002-5416-1956 